\title{
Optical measurements of atmospheric particles from airborne platforms: in situ and remote sensing instruments for balloons and aircrafts
}

\author{
Carlo Buontempo $\left({ }^{1}\right)$, Francesco Cairo $\left({ }^{1}\right)$, Guido Di Donfrancesco $\left({ }^{2}\right)$, Roberto Morbidini $\left({ }^{1}\right)$, \\ Maurizio Viterbini $\left({ }^{1}\right)$ and Alberto Adriani $\left({ }^{3}\right)$ \\ (') Istituto di Scienze dell'Atmosfera e del Clima (ISAC), CNR, Roma, Italy \\ ${ }^{(2)}$ ENEA C.R. Casaccia, Divisione Ambiente Globale e Mediterraneo, \\ Sezione Clima, Santa Maria di Galeria (RM), Italy \\ $\left({ }^{3}\right)$ Istituto di Fisica dello Spazio Interplanetario (IFSI), CNR, Roma, Italy
}

\begin{abstract}
Multiwavelength laser backscattersondes (MAS) have been widely used from a variety of airborne platforms for in situ measurements of optical properties of clouds and atmospheric particulate as well as their phase and composition. Recently, a new miniaturized LIDAR (MULID) has been developed using state-of-art technology for balloon borne profiling of the same quantities. A description of the two instruments, a survey of preliminary results obtained during test flights and indications for future use are given.
\end{abstract}

Key words remote sensing - LIDAR - backscattersonde-aerosol

\section{Introduction}

In response to the changing needs of atmospheric aerosol and cloud research, two new lightweight optical instruments have been developed: a microlidar (MULID) and a Multiwavelength Aerosol Scattersonde (MAS) (Adriani et al., 1999; Buontempo et al., 2003). Primary goal of MAS was its use on board of M55 stratospheric airplane Geophysica (Stefanutti et al., 1999) in the context of polar campaigns. Although Polar Stratospheric Clouds (PSCs)

Mailing address: Dr. Francesco Cairo, Istituto di Scienze dell'Atmosfera e del Clima (ISAC), CNR, Via del Fosso del Cavaliere 100, 00133 Roma, Italy; e-mail: f.cairo@isac.cnr.it and stratospheric aerosol are known to play an important role in ozone depletion (Crutzen and Arnold, 1986), our understanding of PSCs is still in progress and many questions remain open. It is crucial to understand, in terms of particles concentration, sizes and optical parameters, (Rosen et al., 1989, 1990) the microphysical processes underlying PSC structure (Adriani et al., 1992).

For these reasons MAS has been extensively used in several polar campaigns in the context of the European Airborne Polar Experiment (APE) (Stefanutti et al., 1999). The incredible amount of valuable information obtained by MAS during these campaigns led us to extend the use of this instrument to other cloud research fields. We focused our interest in tropical region mainly because of the increasing importance of the equatorial stratosphere in climate modeling. The use of a scattersonde in the Tropical Tropopause Layer (TTL) could help our understanding of formation, growth and decay of ultrathin tropical 
cirrus. Such faint clouds are supposed to play an important role in radiation balance of the tropical atmosphere because of their extension and ubiquity. Moreover the Sub Visual Cirrus (SVCs) appeared to be directly linked to dehydration of the stratosphere with tropical tropopause playing the role of cold trap for ascending air (Holton et al., 1995). Such thin cirrus layers are composed of ice particles and can exist in regions where there is balance between ascent rate, mixing ratio and gravity evaporation. Moreover, as in the polar stratosphere, the presence of a solid substrate could play an important role in the heterogeneous reaction of ozone depletion (Molina, 1991). The experimental device has been completely rebuilt to better fulfill its tropical task.

Although in situ measurements are extremely important to study the life cycle of cirrus cloud, knowledge of the atmospheric environment below the cloud level is important as well. For this reason we have developed a remote sensing instrument. The lightweight microlidar (MULID) designed to be used onboard of stratospheric balloon is, as far as we know, the first balloon borne microlidar ever realized, although in the last two decades many airborne lidars have been developed (Spinhirne, 1982) .

Both the instruments have the capability to measure the depolarized signal backscattered by cloud particles. Such measurements are easily linked to phase and dimension of the scattering particles (Spinhirne et al., 1983).

\section{Optical and mechanical layout}

MAS and MULID use a similar layout, but they mainly differ in the acquisition mechanism and software. For both of them light source is a solid state laser while an optical device is used to collect the backscattered light and to divide it according to its wavelength. Table I shows the optical and electronic characteristics of the two systems.

\section{1. $M A S$}

The system was designed to sample air parcels located few meters away from the plane,
Table I. Optical and electronic characteristics of MULID and MAS instruments.

\begin{tabular}{ll}
\hline \hline MULID & \\
Filter bandwidth & $5 \mathrm{~nm}$ \\
Wavelength & $532 \mathrm{~nm}$ \\
Telescope focal length & $2000 \mathrm{~mm}$ \\
Telescope diameter & $210 \mathrm{~mm}$ \\
Laser peak energy & $1.5 \mu \mathrm{J}$ \\
Pulse repetition rate & $1.1 \mathrm{kHz}$ \\
Total weight & $10 \mathrm{~kg}$ \\
Photo-detector QE @532 nm & $12 \%$ \\
MAS & \\
Filter bandwidth 532 nm & $5 \mathrm{~nm}$ \\
Wavelength & $532 \mathrm{~nm}$ \\
Telescope focal length & $50 \mathrm{~mm}$ \\
Telescope diameter & $48 \mathrm{~mm}$ \\
Laser peak energy & $1.5 \mu \mathrm{J}$ \\
Pulse repetition rate & $1 \mathrm{kHz}$ \\
Total weight & $50 \mathrm{~kg}$ \\
Photo-detector QE @522 nm & $12 \%$ \\
\hline
\end{tabular}

and consequently adopts a $50 \mathrm{~mm}$ common camera lens capable to detect the backscattering signal. It uses three different wavelength $(532,1064$, and $1550 \mathrm{~nm}$ ) to better constrain microphysical and optical characteristics of the sampled particles. The SNR of the system is directly related the ratio between the Field of View (FoV) and the laser beam divergence. A large FoV induces an increase in the background light while a FoV reduction permits the laser beam to complete overlap the FoV only at a very far distance from the instruments, so reducing the «near field» power signal. A $50 \mu$ wide pin hole (FoV $1 \mathrm{mrad}$ ) has been tested initially but after some test flights it clearly appeared that the drawbacks were not balanced by the reduction of the background noise. It was finally set at $3 \mathrm{mrad}$.

A photocell device was used to control a metal shutter at the focal point of the system, to avoid photodetectors damages induced by direct sun light exposure. The incoming beam is collimated and then divided according to its wavelength thanks to a beam splitter. Polarizing cubes 


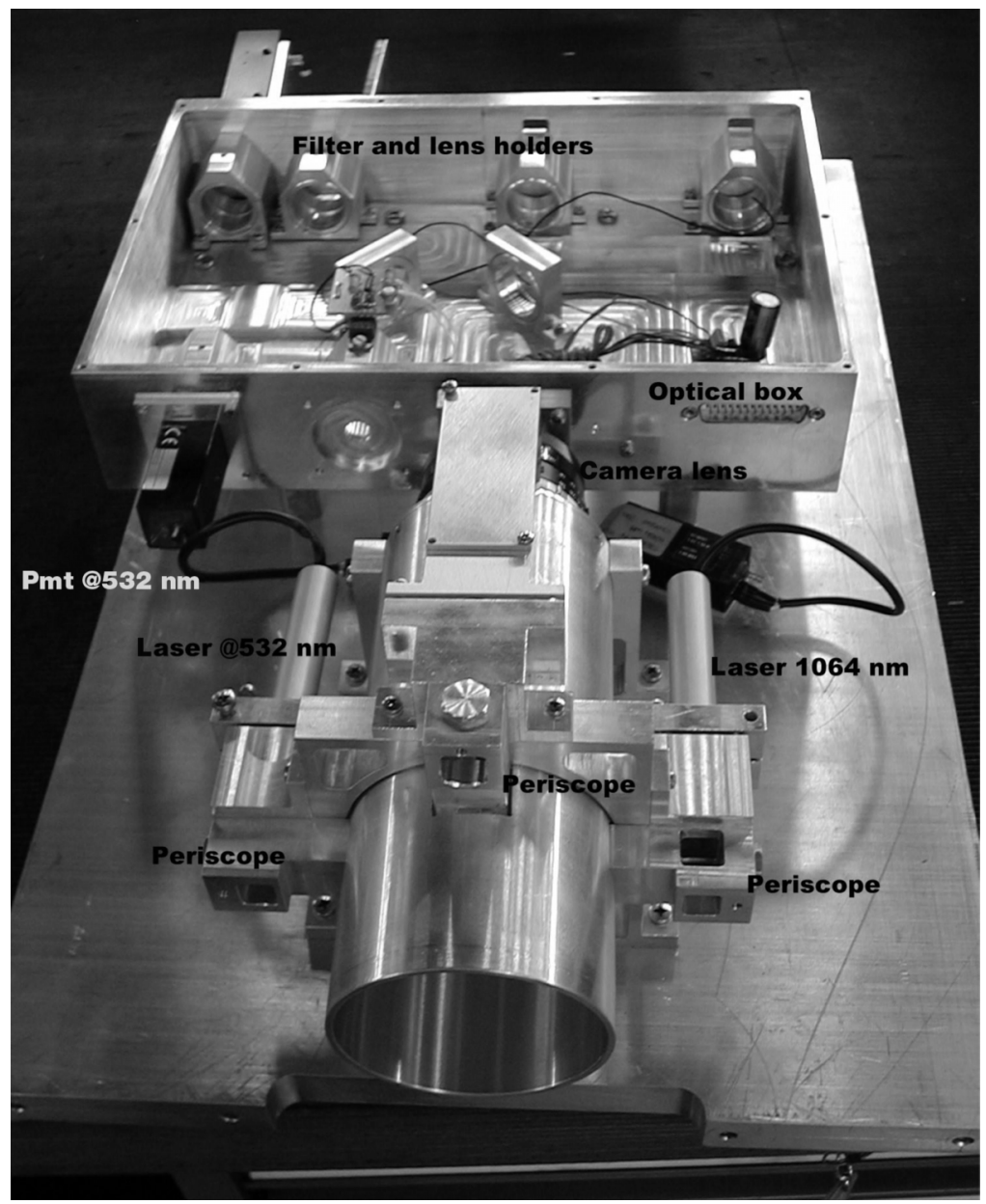

Fig. 1. MAS at the end of mechanical buildup. From above note the optical box with the supports for all the optics, the telescope with the two lasers on its side and the periscope.

will split it again according to its polarization. We adopted photodiode devices for IR channels and photo multipliers for the visible ones.

The system uses interferential filter to select the requested wavelength. A narrow filter improves the SNR but needs a more accurate thermalization to prevent the transmission peak drifting too far away from the required wavelength. MAS flies on board of Geophysica M55 a stratospheric research airplane thus it is subjected to abrupt changes in temperature. Such thermal shocks alter the laser cavity dimensions and consequently the output power. The system adopts an amplified photodiode to monitor laser beam intensity. We realized that not taking into consideration such sharp variation (50-70\%) of laser power, leads to a misleading result. The instrument is divided into two parts mounted one above the other inside a fibreglass sandwich shield. The optical box is located on top, below the acquisition hardware (fig. 1). MAS has four different channels of acquisition: one 


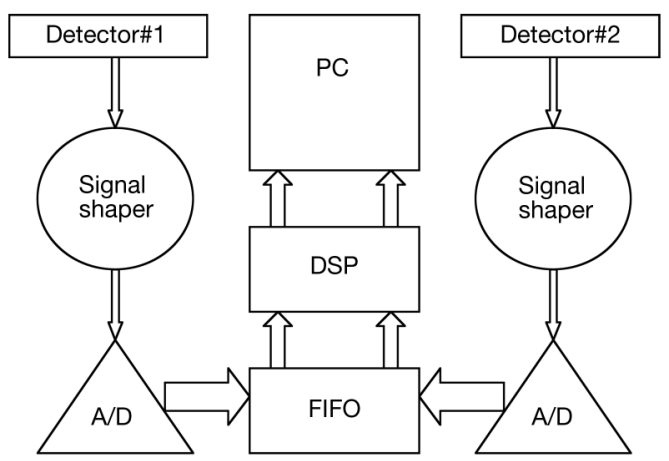

Fig. 2. MAS, electronic data stream: schematic layout.

per wavelength and one for the housekeeping data. Various stages of data elaboration can be identified by following data stream from the photo detector toward its solid state storage on the $\mathrm{PC}$ drive as shown in fig. 2:

- A pulse shaper used to maximise the electronic dynamic.

- An A/D converter that digitalize the profile.

- A First In First Out device (FIFO) used to accumulate data during the pre-trigger phase.

- A Digital Signal Processor (DSP) used to integrate the profile over a preset repetition interval.

- The acquisition PC.

At the end of the flight, after landing, recorded data are retrieved using PC serial ports.

\subsection{MULID}

The system is based on a $1.5 \mu \mathrm{J}$ laser that operates at a repetition rate of $1 \mathrm{kHz}$. A picture describing the instrument is shown in fig. 3. Laser operates at $532 \mathrm{~nm}$, the well known neodinium-YAG source. Sensors are Hamamatsu 5783p photo-multipliers modules used in photon counting mode. Optical and electronic characteristics of the system are shown in table I. The whole system has been embedded in an insulating polyurethane shield to reduce heat exchange with external environment and externally covered by a reflecting Mylar microfilm. Signal acquisition is based on Texas Instrument DSP, characterized by a very low power consumption. The DSP has been piloted by a MS-DOS based PC104.

A pack of commercial alkaline batteries was mounted as power supply during short duration flights while Lithium batteries were preferred for long duration flights. To operate on a balloon platform, MULID needs to be lightweight, compact and very robust. The results were well above our best expectations. After a shock landing, in one of our test flights, major damage was reported only in the telescope while impressively optical and electronic devices were still operating thanks to a shock absorbing carbon fibre structure. The optical layout of MULID is similar to

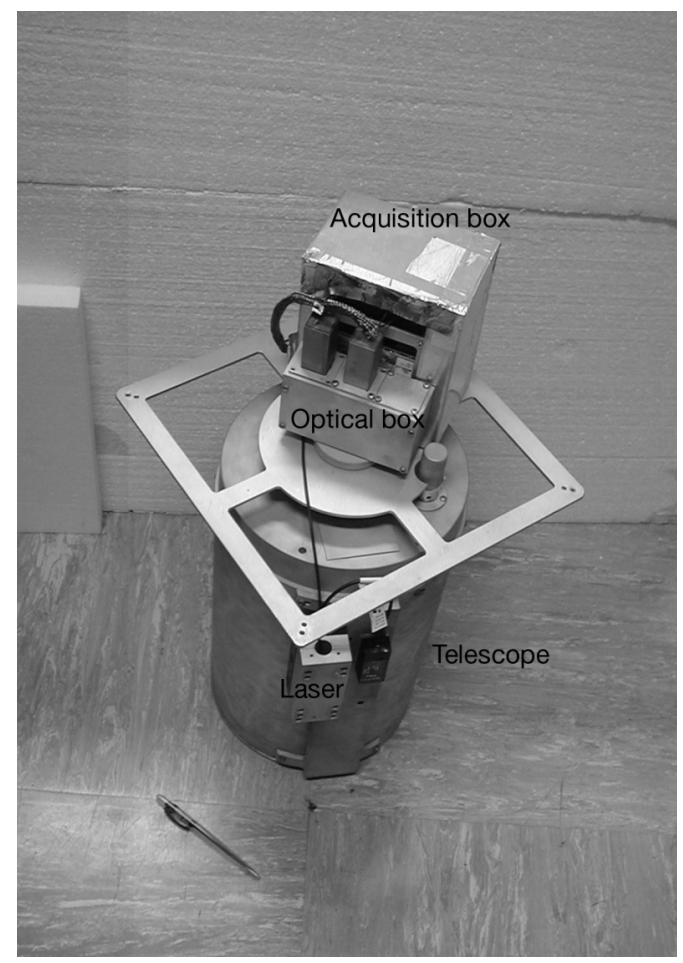

Fig. 3. MULID during the assembling phase. The squared structure on the top is necessary to sustain the carbon fiber and mylar reflecting shield. The optical box is mounted near the focal point of the telescope. 
the MAS one but it operates only at one wavelength instead of the three used by MAS. The system operates in photon counting mode to increase SNR, for this reason data stream is slightly different from the MAS one. The signal coming from photon-multipliers is sent to an electronic device that eliminates low amplitude events caused by thermal background, and reshapes the other pulses to a TTL level (discriminator). The DSP receives the signal and uses its memory as a shift register for the profile. In order to avoid multiple counts of a single event, a synchronization between photon pulse and DSP trigger is performed at this stage. DSP sums the whole profile bin by bin over an interval set by the user (normally $30 \mathrm{~s}$ ). After the integration period the summed profile is sent to the $\mathrm{PC}$ where is stored on a solid state memory (2 MB). The use of a $40 \mathrm{MHz}$ DSP allows to average profiles at the laser firing frequency $(1.1 \mathrm{kHz})$.

Several problems are related to the use of such an accurate optical system in an environment as hard as the stratosphere. The photonmultipliers are not designed for such low pressure use: when external pressure falls below 50 $\mathrm{mb}$, dielectric breakdown occurs in the air between dinodes. Such electric discharges damage PMTs permanently. To fix the problem the photon multiplier windows were treated with a varnish to create a multilayer external coating that avoids air loss during ascent.

\section{Data inversion}

For both the instruments it is necessary to invert and calibrate the signal to obtain atmospheric data. Most of the algorithms used to invert LIDAR data assume the signal to be pure molecular at a certain height. For MULID the procedure is straightforward although the molecular point is located in the close range. Hereafter we analyse the way we invert MULID data and then we will describe how to extend this technique to an in situ instrument that does not have a pure molecular point per profile. For a ground based LIDAR the signal equation is the following:

$$
S(r)=A \cdot \frac{1}{r^{2}} \cdot\left(\beta_{m}(r)+\beta_{a}(r)\right) \cdot \int_{0}^{r} \sigma(r) d r
$$

where $A$ is a constant related to overall efficiency of the system, $r$ is the distance from the observer; $S$ is the signal; the two terms in brackets represent the molecular and the aerosolic contribution to the scattering respectively, the integral term represents the signal extinction. In a ground based LIDAR the inversion is performed assuming as pure molecular the signal coming from the upper stratosphere and then using an iterative method to calculate the integral term in the eq. (3.1), taking for granted the relation between molecular backscattering and extinction. In our case the inversion algorithm is calibrated in the short range.

During balloon ascent, gondola housekeeping data allow us to calculate the density profile. We use such profile to calculate molecular contribution at every height. To calculate aerosol scattering ratio $(R)$ we postulate that a few kilometres above the tropopause the signal has to be almost pure molecular so that

$$
R\left(Z_{\text {tropopause }}+\delta Z\right)=\frac{\left(\beta_{m}(r)+\beta_{a}(r)\right)}{\beta_{m}(r)} \approx 1.1 .
$$

This is a reasonable assumption since it is well known that the aerosol profile exhibits a minimum above the tropopause (Jager et al., 1995).

The range corrected signal is calibrated using this assumption with an iterative method calculating the whole profile. At this stage the cloud extinction in the signal profile is being considered. A problem exists for those profiles that do not include any stratospheric point in the profile as for instance those obtained during tropospheric ascent. In these cases we have calibrated the profile considering valid the calibration of the first stratospheric profile. Because of the insulator shield, radiative cooling inside the microlidar is not too fast, as good approximation we assume that only minor laser temperature variation happens during the balloon fast ascent. It is possible to check such approximation looking at the surface reflectivity, that is supposed to be almost constant.

For an in situ probe the inversion technique is even more complicated. MAS does not acquire a profile as MULID does, it uses the backscattered value integrated on the whole profile. For the increase of the SNR of the in- 
strument, the software subtracts the signal averaged over a region where the beam is out of the telescope FoV, from the backscattered integrated peak value. For MAS, lasers are mounted on the telescope fig. 1, where outside air is very likely to rapidly cool the instrument. Taking into account laser power variation has a first order importance for MAS. We use laser monitor to correct such variations and performed several lab tests to calibrate laser meter output to actual laser beam power and we are absolutely confident in the accuracy of our calibration technique.

When spurious laser effects are eliminated from the signal, airplane data stream is used to calculate the molecular contribution to the scattering and then a scattering ratio $R$ value is calculated. To calibrate the $R$ value we assume the value of the scattering ratio is known at some particular height.

We normally use $19000 \mathrm{~m}$ as a reference for $R=1.1$ scattering ratio. For MAS it is sufficient to have at least a couple of points in the stratosphere to calibrate the whole flight because of the real-time laser power calibration.
Particular attention was paid to the computer synchronization for both instruments. The clock of the acquisition PC has a different speed from the geographical data stream one. We have observed CPU clock drift as big as 700 $\mathrm{s}$ in a $4000 \mathrm{~s}$ flight span. This is surely due to the hazardous condition encountered by the system during the flight. Not to synchronize the clocks leads to erroneous results.

\section{Results}

In this paper we present some of the first results of both systems. Data have to be considered as preliminary. Both of them refer to two technical flights. For MULID we present (fig. 4) data from a tropical flight performed from Bauru (Brazil) on February 23rd 2003. Color represents the scattering ratio that is supposed to be 1 in absence of cloud. Three distinct feature are visible on the plot. At the bottom it is possible to notice a shallow layer where $R$ is clearly above 1 . This layer represents the nocturnal residual layer. The increase in scattering

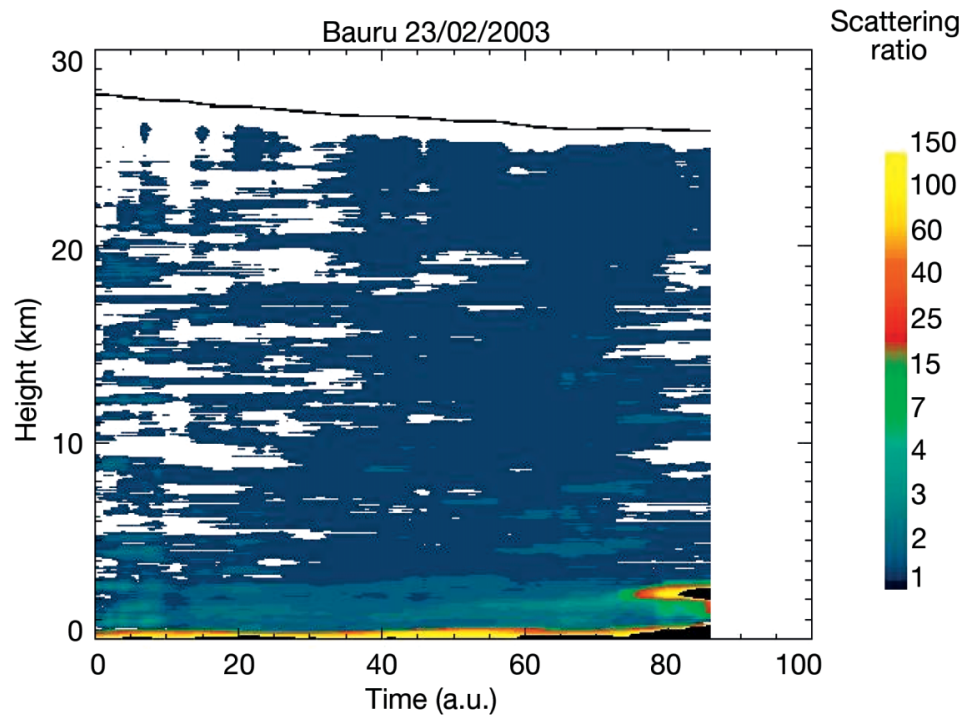

Fig. 4. MULID profile of backscattering ratio obtained in Brazil during a night time short duration flight. PBL is detected as a shallow area near the ground where scattering is above 1 . A cloud is detected at the end of acquisition period. 


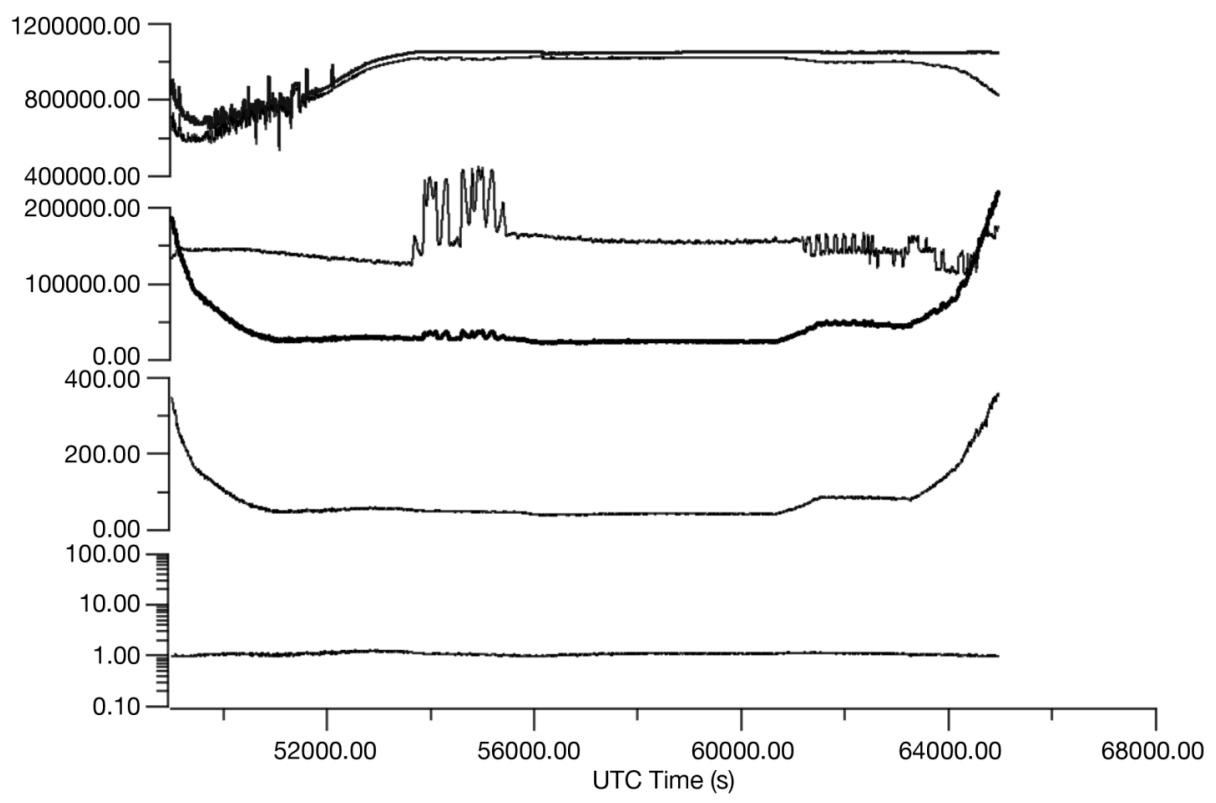

Fig. 5. MAS data stream. From above signal of the backscattered peak and background signal, laser monitor line, actual signal (peak-background) not yet corrected for the laser power variations, corrected signal and finally calibrated scattering ratio. In this case the absence of clouds is evidenced by the $R$ constancy.

ratio is mainly due to high concentration dust and cloud droplet often found below this mixing layer. At the bottom, on the right, there is a single cloud layer similar to a low level stratocumulus. Finally, it is possible to notice the ground reflectance as a sharp increase in scattering ratio.

We have not included in the plot the data coming from places where the laser beam is not completely inside the telescope FoV. It has to be mentioned that to increase SNR we voluntary reduced spatial and temporal resolution by the use of a running average, to $150 \mathrm{~m}$ and 5 min respectively.

For MAS we present data from a flight performed on February 6th 2003 from Kiruna (Sweden) during the EUPLEX campaign. In the plot (fig. 5) it is possible to follow the complete sequence of calibration operated on the raw data. From the top you can notice the peak signal and the background. The difference between the two leads to the fourth line (second of the second plot box) that represents the signal not yet normalized by laser power variations. Those laser variations are indicated by the third line (first of the second plot box). Taking into account the laser effect we have the corrected signal plot. If we calibrate the signal above tropopause as mentioned in the previous section we obtain $R$ signal.

\section{Conclusions}

We have presented two innovative lightweight optical instruments. MULID is the first balloon borne microlidar ever built. As our preliminary results demonstrates, it will be very useful in the tropics. At low latitude accurate observation of microphysical properties of cirrus clouds are very important: both for cirrus modeling and for the comprehension of the stratosphere to troposphere exchange process (Danielsen, 1993).

MULID will provide an interesting dataset regarding faint tropical cirrus clouds. Also ac- 
curate in situ measurements are basic for the understanding of cloud microphysics.

MAS represents the state of the art backscatter sonde. It works, both at night and day, at three different wavelengths in the two polarizations. Here we have presented only the data of the visible channel.

The backscattering at three wavelengths and the color ratio will help us to better understand the formation of PSCs and cirrus cloud.

\section{REFERENCES}

Adriani, A., T. Deshler, G.P. GobBi, B.J. Johnson and G. Di Donfrancesco (1992): Polar stratospheric clouds over McMurdo, antartica, during 1991 spring: LIDAR and particle counter measurements, Geophys. Res. Lett., 19, 1755-1758.

Adriani, A., F. Cairo, M. Viterbini, S. Mandolini, L. Pulvirenti and G. Di Donfrancesco (1999): Multiwavelength Aerosol Scatterometer for airborne experiments to study the stratospheric particle optical properties, J. Atmos. Oceanic Technol., 16, 1329-1336.

Buontempo, C., F. Cairo, M. Snels, G. Di Donfrancesco, R. Morbidini, M. Viterbini, F. CARdillo, A. Adriani and M. MORIONI (2003): The detection of clouds and aerosols with the backscattersonde mas during the M55 Geophysica campaign for envisat validation, in Proceedings of the 16th ESA Symposium on European Rocket and Balloon Programmes and Related Research, ESA SP-530, August 2003.
Crutzen, P J. and F. ARnold (1986): Nitric acid cloud formation in the cold Antartic stratosphere: a major cause for the springtime ozone hole, Nature, 324, p. 651.

DANIELSEN, E.F. (1993): In situ evidence of rapid, vertical, irreversible transport of lower tropospheric air into the lower tropical stratosphere by convective cloud turrets and by larger-scale upwellin in tropical cyclones, $J$. Geophys. Res., 98, 8665-8681.

Holton, J.R., P.H. Haynes, M.E. McIntyre, A.R. DouGLASS, R.B. RoOD and L. PFISTER (1995): Stratospheretroposphere exchange, Rev. Geophys., 33 (4), 403-440.

Horst, J., T. DeshleR and D.J. HofMAnN (1995): Midlatitude LIDAR backscatter conversions basedon balloonborne aerosol measurements, Geophys. Res. Lett., 11, 1729-1732.

MolinA, M.J. (1991): Heterogeneous chemistry on polar stratospheric clouds, Atmos. Environ., 25A, 2535-2537.

Rosen, J.M., S.J. Oltmans and W.F. Evans (1989): Balloon borne observation of PSCs, ozone, and Nitric acid in the north polar Vortex, Geophys. Res. Lett., 16, 791-794.

Rosen, J.M., N.T. KJOME and S.J. Oltmans (1990): Observation of of backscattere, particles concentration and frost point in north polar vortex stratospheric clouds, Geophys. Res. Lett., 17, 1271-1274.

SpinhiRne, J.D., M.Z. HANSEN and L.O. CAUdill (1982): Cloud top remote sensing by airborne LIDAR, Appl. Opt., 21, 1564-1571.

SPINHIRNE, J.D., M.Z. HANSEN and J. Simpson (1983): The structure and phase of clouds tops as observed by polarization LIDAR, J. Appl. Meteorol., 22, 1319-1331.

Stefanutti, L., A.R. MacKenzie, S. Balestri, V. KhattaTOV, G. FIOCCO, E. Kyro and T. PETER (1999): Airborne Polar Experiment-Polar Ozone, Leewaves, Chemistry, And Transport (APE-POLLECAT): rationale, road map and summary of measurements, J. Geophys. Res., 104, 23,941-23,959. 